\title{
Persistent Organochlorine Exposure and Pregnancy Loss: A Prospective Cohort Study
}

\author{
Anna Z. Pollack ${ }^{1}$, Germaine M. Buck Louis ${ }^{1}$, Courtney D. Lynch ${ }^{2}$, Paul J. Kostyniak ${ }^{3}$ \\ ${ }^{1}$ Epidemiology Branch, Eunice Kennedy Shriver National Institute of Child Health and Human Development, Bethesda, USA; \\ ${ }^{2}$ Division of Epidemiology \& Department of Obstetrics and Gynecology, Ohio State University, Columbus, USA; ${ }^{3}$ Department of \\ Pharmacology and Toxicology, School of Medicine and Biomedical Sciences, University of New York, Buffalo, USA. \\ Email: pollacka@mail.nih.gov
}

Received March 23 ${ }^{\text {rd }}, 2011$; revised May $6^{\text {th }}, 2011$; accepted June $21^{\text {st }}, 2011$.

\begin{abstract}
Polychlorinated biphenyls (PCBs) and 1,1-dichloro-2,2-bis(p-chlorophenyl)ethylene (DDE) are suspected reproductive toxicants. We assessed serum concentration of 76 PCB congeners, DDE, and risk of human chorionic gonadotropin confirmed pregnancy loss among 79 women followed for up to 12 menstrual cycles or until pregnancy. 55 women had live births, 14 experienced pregnancy losses, and 10 did not achieve pregnancy. PCBs and DDE were quantified using gas chromatography with electron capture. PCBs were grouped a priori by biologic activity. Cox proportional hazard regression adjusting for age (categorized 24 - 29, 30 - 34) and average standardized alcohol and cigarette intake (continuous) was used to estimate hazard ratios (HR) of pregnancy loss. Estrogenic PCBs (HR $=1.66,95 \%$ CI: 0.68, 4.02), anti-estrogenic PCBs $(H R=0.10,95 \%$ CI: <0.01, 67.07) and DDE $(H R=1.43,95 \%$ CI: 0.45, 4.52) were not statistically significantly associated with pregnancy loss. Our results provide some signal that estrogenic and antiestrogenic PCBs may be differentially associated with pregnancy loss. Further research is needed to elucidate these associations.
\end{abstract}

Keywords: 1,Dichloro-2,2-Bis(P-Chlorophenyl)Ethylene, Fecundity, Organochlorine, Polychlorinated Biphenyl, Pregnancy Loss

\section{Introduction}

Fecundity is defined as the biologic capacity for reproduction [1] and can be conceptualized to include impairments such as conception delay, infertility, or pregnancy loss. Fecundity is an essential aspect when assessing potential reproductive or developmental toxicity of environmental agents. Exposures adversely impacting fecundity can be identified by estimating differences in the time-to-pregnancy among exposed women relative to unexposed women [2] or in estimating the risk of pregnancy loss [3]. There has been speculation that a global reduction in human fecundity is underway [4]. Available evidence suggests that environmental agents, particularly the endocrine disrupting class of chemicals, may contribute to this decline [5].

Recent reviews have summarized the available evidence regarding the reproductive and/or developmental toxicity (RADT) of persistent organochlorines such as polychlorinated biphenyls (PCBs) and 1,1-dichloro-2,2bis(p-chlorophenyl)ethylene (DDE) [6-8]. Past research has attempted to assess the potential RADT of polyhalo- genated aromatic hydrocarbons in relation to human fecundity, but often has relied upon proxy exposure measurements such as PCB contaminated fish consumption [9,10], occupation [11], residence [12], or quantified serum or plasma concentrations often collected years following reproductive outcomes $[13,14]$. Estimating the effect of environmental chemical exposure and pregnancy loss is challenging for many reasons, including the high proportion of losses occurring prior to (woman/ clinical) recognition and the competing risk of infertility, in that women who do not conceive are not at risk of pregnancy loss. Prospective cohort designs with preconception recruitment of women or couples and with biomonitoring of human chorionic gonadaotropin (hCG) pregnancy remains the gold standard for assessing environmental RADT, particularly those occurring in the sensitive periconceptional window $[15,16]$. While challenging, the utility and feasibility of such approaches have been established [17].

Spontaneous pregnancy loss is a sensitive endpoint indicative of impaired fecundity. In relation to DDT exposure, pregnancy loss has been assessed by questionnaire 
with no effect [14] while a positive association was found with an hCG biomarker of pregnancy loss [18]. Repeated miscarriage was not related to organochlorine exposure when pregnancy loss was assessed by maternal recall $[19,20]$. Prospectively observed pregnancy loss and organochlorine exposure have yet to be assessed using biomarkers of PCB, DDE, and pregnancy. The paucity of literature on the potential influence of environmental factors on fecundity impairments, coupled with the growing consensus that such an effect is present motivated the current study. We assessed the relation between PCB and DDE concentrations and risk of hCG pregnancy loss in a cohort of women recruited prior to conception and followed until pregnant or through 12 menstrual cycles at risk for pregnancy.

\section{Methods}

The study cohort was recruited from a referent cohort comprising 2,637 women aged 18 - 44 years who participated in a population-based angler cohort study whose aim was to assess species specific fish consumption and knowledge of consumption advisories. Specifically, women who stated in 1991 that they were either considering or undecided about future pregnancies were re-contacted in 1996 - 1997 and asked to participate in a prospective pregnancy study, providing they were $\leq 40$ years of age, had no physician-diagnosed infertility and were planning to begin trying to become pregnant in the next six months. Among the 244 eligible women, 113 (46\%) were recruited, of which 14 were found to be pregnant at or immediately following enrollment, leaving 99 women in the study cohort. An additional 20 women withdrew from the study without an observed pregnancy. Of the 79 women completing follow-up, 69 (87\%) became pregnant, of which $55(80 \%)$ had live births and $14(20 \%)$ experienced pregnancy losses. Ten $(13 \%)$ women did not achieve pregnancy (i.e., infertile). The study was approved by the Institutional Review Board for the School of Medicine and Biomedical Sciences at the University at Buffalo, State of New York; all women provided informed consent prior to their participation.

Women participated in a baseline interview administered by a research registered nurse and were instructed in the accurate use of the Clearblue Easy ${ }^{\mathrm{TM}}$ home pregnancy test, capable of detecting $\leq 50 \mathrm{mIU} / \mathrm{ml}$ of hCG (per manufacturer) in urine on the date of expected menses. Clearblue Easy is a digital pregnancy test kit that displays "pregnant", "not pregnant", or "error" for simple interpretation. This pregnancy test is one of the most sensitive and accurate presently available [21]. Participants were instructed to use pregnancy tests on the first day menses was expected and one week later, regardless of the first result, and to complete daily diaries while trying to become pregnant to obtain information on menstruation, sexual intercourse and lifestyle exposures that may adversely affect fecundity, including pregnancy loss (i.e., cigarette smoking and consumption of caffeine and alcohol). Reminder telephone calls were placed by the research nurse when diary cards were a week late. A non-fasting blood sample $(10 \mathrm{ml})$ was collected at the baseline interview by the research nurse utilizing venipuncture collection equipment determined to be free of the chemical exposures of interest in the study.

Serum samples were analyzed by the Toxicology Research Center at the University at Buffalo (Buffalo, NY, USA), for 76 congeners (64 single and 12 di-eluting congeners) using gas chromatography with electron capture detection as previously described [22,23]. Briefly, ten samples were run in each batch, including four controls consisting of one reagent blank, one matrix blank, one quality control sample and one duplicate sample [22]. PCB congeners were categorized a priori by purported biologic activity and summed $\left(\sum\right)$ into four groupings: 1) total PCBs or the $\sum$ of all measured congeners; 2) $\sum$ estrogenic congeners (\# 4_10,5_8, 15_17, 18, 31, 44, 47, 48, 52, 70, 99, 101, 136, 153, 188); 3 ) $\sum$ anti-estrogenic congeners (\# 77_110, 105, 114, 126, 156_171, 169); and 4) $\sum$ other $\mathrm{PCB}$ congeners (\#6, 7 9, 16 32, 19, 22, $24 \_27,25,28,33,40,42,45,50,55,59,60,64,66 \_95$, 74, 81_87, 82, 94, 97, 118, 128, 129, 132, 134, 135, 138, $141,147,149,151,157 \_200,163,167,170,172,174$, $176,177,179,180,181,183,185,187,189,190,194$, 195, 196_203, 205, 206) $[24,25]$. PCB and DDE values were corrected only for recovery; no substitution of values below the laboratory limits of detection were made to avoid introducing potential bias [26,27]. We did not adjust PCBs or DDE for serum lipids, given the absence of evidence supporting a causal relation between serum lipids and pregnancy loss [28]. Serum PCB and DDE concentrations are expressed as nanogram/gram (ng/g) serum, which is equivalent to parts per billion. Total lipids (TL) were quantified using enzymatic methods as a function of total cholesterol (TC), free cholesterol (FC), triglycerides (TG) and phospholipids (PL) as:

$\mathrm{TL}=1.677(\mathrm{TC}-\mathrm{FC})+\mathrm{FC}+\mathrm{TG}+\mathrm{PL}$, and were expressed in $\mathrm{mg} / \mathrm{dl}$ [29].

The primary outcome of interest was pregnancy loss. Early pregnancy loss was defined as a positive pregnancy test followed by a negative test or by clinical confirmation of pregnancy followed by clinical pregnancy loss, which was defined as loss following clinical confirmation of pregnancy. Clinical pregnancy losses were selfreported, though all women who had hCG confirmed pregnancies were followed for approximately eight weeks' gestation. All clinical losses occurred prior to 20 weeks' gestation as reported by women. In our analyses, total 
pregnancy loss included early and clinical loss.

Lifestyle covariates (caffeine, alcohol, and cigarettes smoked) were reported daily using the diary and were, subsequently, standardized to a 28-day cycle to account for the intra- and inter-woman variability in menses. Number of caffeinated (coffee, tea, or soda) and alcoholic drinks and number cigarettes smoked per day were recorded on the daily diary. Specifically, we summed the woman's reported consumption over her menstrual cycle (which may be longer or shorter than 28 days), multiplied it by 28 and then divided by the number of days in her cycle. Failing to standardize lifestyle exposures could make it seem that women with shorter menstrual cycles consumed less, because they had less opportunity for consumption at each cycle; conversely, that women with longer cycles consumed more given their longer length of follow-up. Caffeine and alcohol consumption and cigarette smoking were selected because of their possible shared detoxification pathway with $\mathrm{PCBs}$ and DDE via cytochrome P450 (CYP450) [30]. High levels of lifestyle covariates could lead to overloading this pathway; alternatively, individuals with high lifestyle covariate levels could have CYP450 pathways that are better suited to detoxification. Confounders were selected based on $a$ priori knowledge.

Descriptive population statistics were tabulated by study outcome variable, and by completion of the study protocol. Pearson's Chi Square and ANOVA $p$-values were calculated to detect statistically significant differences $(p<0.05)$ between study outcome and completion of the study protocol.

Although all participants provided blood samples, some women did not have sufficient sample to quantify all congeners $(\approx 10 \%)$ or individual lipid (30\%) components necessitating the use of multiple imputation. Cigarette and alcohol consumption were imputed for two individuals with insufficient daily diary data, but who completed follow-up. Multiple imputation was used to estimate the missing values to avoid potential biases associated with mean or median imputation, or complete case analysis [31-33] using Imputation by Chained Equations (ICE) in STATA version 9 (StataCorp, College Station, TX).

Cox proportional hazard regression was used to estimate hazard ratios (HRs) of pregnancy loss (early and clinical losses combined) when restricting to women achieving pregnancy while under observation. Censoring occurred after 20 weeks of pregnancy. We estimated the hazard of pregnancy loss for PCB and DDE concentrations when left as continuous or categorical exposures. Models for pregnancy loss were run separately for total PCBs, estrogenic PCBs, anti-estrogenic PCBs, other PCBs, and DDE. Additionally, estrogenic PCBs, anti- estrogenic PCBs, other PCBs, and DDE were run together as continuous variables in one model, to determine if the results from the individual models were altered, perhaps due to a competing mechanism of action. We presented all models with and without adjustment for age (categorized as 24 - 29 and 30 - 34) and average standardized alcohol and cigarette intake over a 28-day menstrual cycle (continuous). We did not include parity as a covariate because it may be on the causal pathway to pregnancy loss, and may induce over adjustment bias [34,35]. Additionally, we emphasize that given our small sample size, our statistical models are not robust to stratifying by parity to further assess this issue. STATA version 9 was used for all analyses.

\section{Results}

All women completed baseline interviews and returned $95 \%$ of expected daily diary cards. Women who withdrew from the study either before achieving pregnancy or completing 12 menstrual cycles with intercourse during the fertile window were not systematically different from women completing the study with regard to age, gravidity, parity, BMI, or smoking, alcohol and cigarette consumption (data not shown). Gravidity was associated with pregnancy loss (Table 1). However, age, body mass index $\left(\mathrm{kg} / \mathrm{m}^{2}\right)$, smoking, alcohol, and cigarette consumption were not statistically associated with pregnancy loss, infertility, or live birth.

Women with a live birth had a broader range of total PCB levels, 2.58 to $14.51 \mathrm{ng} / \mathrm{g}$ serum, compared to women with a pregnancy loss, 3.89 to $9.09 \mathrm{ng} / \mathrm{g}$ serum (Table 2). Estrogenic PCBs were similarly distributed, and women with a live birth ranged from a minimum of 0.07 to a maximum of $9.19 \mathrm{ng} / \mathrm{g}$ serum, whereas women with a pregnancy loss ranged between 1.63 to $4.52 \mathrm{ng} / \mathrm{g}$ serum. Women with a live birth had a greater range of DDE levels, from $<0.01$ to $3.37 \mathrm{ng} / \mathrm{g}$ serum, compared to those with a pregnancy loss, from 0.41 to $1.63 \mathrm{ng} / \mathrm{g}$ serum. Anti-estrogenic PCBs and other PCBs similarly had a slightly wider range among women with a live birth than women with a pregnancy loss (Table 2).

No statistically significant differences in PCB or DDE concentrations were observed by outcome (Table 2). Total PCBs were lowest among women who did not achieve pregnancy over 12 prospectively observed menstrual cycles (4.62 ng/g serum), and highest among women with a live birth $(5.26 \mathrm{ng} / \mathrm{g}$ serum), although this difference was not statistically significant. Median estrogenic PCB levels were similar among women who had a live birth $(2.20 \mathrm{ng} / \mathrm{g}$ serum $)$ and those who had a pregnancy loss (2.16 ng/g serum). DDE was marginally higher among women with a pregnancy loss (1.06 ng/g 
Table 1. Sociodemographic characteristics of cohort by study outcome $(n=79)$.

\begin{tabular}{|c|c|c|c|c|}
\hline Characteristic & Live Birth n (\%) & Pregnancy Loss n (\%) & Infertile n (\%) & $\mathrm{P}$ value \\
\hline & $55(70)$ & $14(17)$ & $10(13)$ & \\
\hline Age (in years) & & & & 0.25 \\
\hline$<30$ & $19(34)$ & $8(57)$ & $5(50)$ & \\
\hline$\geq 30$ & $36(65)$ & $6(43)$ & $5(50)$ & \\
\hline Gravidity (\# live births) & & & & 0.01 \\
\hline 0 & $10(18)$ & $6(43)$ & $6(75)$ & \\
\hline 1 & $36(65)$ & $5(36)$ & $1(12)$ & \\
\hline $2+$ & $9(16)$ & $3(21)$ & $1(12)$ & \\
\hline Parity (\# pregnancies) & & & & 0.10 \\
\hline 0 & $8(14)$ & $5(36)$ & $5(50)$ & \\
\hline 1 & $24(44)$ & $4(28)$ & $3(30)$ & \\
\hline $2+$ & $23(42)$ & $5(36)$ & $2(20)$ & \\
\hline Body mass index $\left(\mathrm{kg} / \mathrm{m}^{2}\right)$ & & & & 0.88 \\
\hline$<25$ & $34(62)$ & $9(64)$ & $7(70)$ & \\
\hline$\geq 25$ & $21(38)$ & $5(36)$ & $3(30)$ & \\
\hline \multicolumn{5}{|c|}{ Mean $\left( \pm \mathrm{SD}^{\mathrm{b}}\right)$} \\
\hline Daily \# cigarettes smoked & $1.9(4.5)$ & $0.4(1.4)$ & $0.4(1.3)$ & 0.28 \\
\hline Daily \# alcoholic beverages & $0.3(0.3)$ & $0.2(0.5)$ & $0.5(0.4)$ & 0.33 \\
\hline Daily \# caffeinated beverages & $1.8(1.5)$ & $0.9(0.9)$ & $1.4(1.2)$ & 0.10 \\
\hline
\end{tabular}

NOTE: Cigarette usage was imputed for 9 women, 9 for alcohol and 9 for caffeine consumption. Gravidity is missing for 2 women. Excludes 20 women who withdrew from study before an outcome. ${ }^{\mathrm{a}} \mathrm{TTP}$, denotes number of menstrual cycles at risk for pregnancy. ${ }^{\mathrm{b}} \mathrm{SD}$, denotes standard deviation.

Table 2. Median serum concentration of polychlorinated biphenyl (PCBs) and 1,1-dichloro-2,2-bis(p-chlorophenyl)ethylene (DDE) concentrations by study outcome $(n=79)$.

\begin{tabular}{cccc}
\hline $\begin{array}{c}\text { Serum Concentration } \\
(\mathrm{ng} / \mathrm{g} \text { serum})\end{array}$ & $\begin{array}{c}\text { Live Birth }(\mathrm{n}=55) \\
\text { Median }(\min , \max )\end{array}$ & $\begin{array}{c}\text { Pregnancy Loss }(\mathrm{n}=14) \\
\text { Median }(\min , \max )\end{array}$ & $\begin{array}{c}\text { Infertile }(\mathrm{n}=10) \\
\text { Median }(\min , \text { max })\end{array}$ \\
\hline Total PCBs & $5.26(2.58,14.51)$ & $5.14(3.89,9.09)$ & $4.62(4.09,11.08)$ \\
Estrogenic PCBs & $2.20(0.07,9.19)$ & $2.16(1.63,4.52)$ & $1.99(0.63,5.99)$ \\
Anti-estrogenic PCBs & $0.20(<0.02,0.68)$ & $0.18(0.03,0.32)$ & $0.22(0.06,0.35)$ \\
Other PCBs & $2.88(1.58,9.07)$ & $2.83(2.04,4.68)$ & $2.47(2.23,4.99)$ \\
DDE & $0.93(<0.01,3.37)$ & $1.06(0.41,1.63)$ & $0.80(0.47,3.59)$ \\
Serum lipids (mg/dL) & $558.35(369.67,881.49)$ & $555.95(324.20,967.13)$ & $537.74(402.55,713.80)$ \\
\hline
\end{tabular}

Note: Cigarette usage was imputed for 9 women, 9 for alcohol and 9 for caffeine consumption. Gravidity is missing for 2 women. Excludes 20 women who withdrew from study before an outcome.

serum) compared to those with a live birth $(0.93 \mathrm{ng} / \mathrm{g}$ serum).

Total PCBs, estrogenic PCBs, anti-estrogenic PCBs and DDE were not statistically significantly associated with the adjusted hazard of pregnancy loss (Table 3).
However, the point estimates were greater than one for total and estrogenic PCBs and DDE and less than one for anti-estrogenic PCBs. Relative to the lowest tertile, the aHR for pregnancy loss among those in the highest DDE tertile was $1.45(95 \% \mathrm{CI}: 0.37,5.70)$. The highest estro- 
Table 3. Serum concentration of polychlorinated biphenyl (PCBs) and 1,1-dichloro-2,2-bis(p-chlorophenyl)ethylene (DDE) concentrations and hazard of pregnancy loss $(n=69)$.

\begin{tabular}{|c|c|c|}
\hline Serum Concentration (ng/g serum) & Unadjusted HR (95\% CI) & Adjusted HR $(95 \% \mathrm{CI})^{\mathrm{a}}$ \\
\hline \multicolumn{3}{|l|}{ Total PCBs } \\
\hline Low $(1.62-5.02)$ & 1.00 (referent) & 1.00 (referent) \\
\hline Medium (5.03 - 5.71) & $1.06(0.28,4.00)$ & $0.98(0.25,3.82)$ \\
\hline $\operatorname{High}(5.72-12.68)$ & $0.98(0.26,3.66)$ & $1.20(0.31,4.67)$ \\
\hline Total PCBs (continuous) & $1.04(0.77,1.50)$ & $1.15(0.84,1.58)$ \\
\hline \multicolumn{3}{|l|}{ Estrogenic PCBs: } \\
\hline Low $(1.49-2.06)$ & 1.00 (referent) & 1.00 (referent) \\
\hline $\operatorname{Med}(2.07-2.28)$ & $0.59(0.16,2.23)$ & $0.60(0.16,2.31)$ \\
\hline $\operatorname{High}(2.29-4.53)$ & $0.75(0.21,2.66)$ & $0.86(0.23,3.11)$ \\
\hline Estrogenic PCBs (continuous) & $1.61(0.64,4.02)$ & $1.66(0.68,4.02)$ \\
\hline \multicolumn{3}{|l|}{ Anti-estrogenic PCBs: } \\
\hline Low $(0.02-0.16)$ & 1.00 (referent) & 1.00 (referent) \\
\hline $\operatorname{Med}(0.17-0.22)$ & $0.70(0.21,2.35)$ & $0.83(0.24,2.85)$ \\
\hline $\operatorname{High}(0.23-0.65)$ & $0.48(0.12,1.97)$ & $0.71(0.16,3.10)$ \\
\hline Anti-estrogenic PCBs (continuous) & $0.03(<0.01,18.79)$ & $0.10(<0.01,67.07)$ \\
\hline \multicolumn{3}{|l|}{ Other PCBs: } \\
\hline Low $(1.97-2.70)$ & 1.00 (referent) & 1.00 (referent) \\
\hline $\operatorname{Med}(2.71-3.11)$ & $1.43(0.35,5.80)$ & $1.61(0.39,6.72)$ \\
\hline $\operatorname{High}(3.12-9.07)$ & $1.25(0.30,5.32)$ & $1.70(0.38,7.49)$ \\
\hline Other PCBs (continuous) & $0.99(0.58,1.70)$ & $1.09(0.66,1.81)$ \\
\hline \multicolumn{3}{|l|}{ DDE } \\
\hline Low $(0.39-0.82)$ & 1.00 (referent) & 1.00 (referent) \\
\hline $\operatorname{Med}(0.83-1.14)$ & $1.07(0.28,4.06)$ & $1.08(0.28,4.21)$ \\
\hline $\operatorname{High}(1.15-3.60)$ & $1.21(0.32,4.59)$ & $1.45(0.37,5.70)$ \\
\hline DDE (continuous) & $1.09(0.38,3.15)$ & $1.43(0.45,4.52)$ \\
\hline
\end{tabular}

NOTE: Estrogenic PCBs includes $\sum$ congeners 4_10, 5 _ 8, $15 \_17,18,31,44,47,48,52,70,99,101,136,153,188$; anti-estrogenic PCBs includes $\sum$ congeners $77110,105,114,126,156$ 171, 169; and other includes $\sum$ PCB congeners 6, 7 9, 16 32, 19, 22, 24 27, 25, 28, 33, 40, 42, 45, 50, 55, 59, 60, 64, 66 95, 74, 81_87, 82, 94, 97, 118, 128, 129, 132, 134, 135, 138, 141, 147, 149, 151, 157_200, 163, 167, 170, 172, 174, 176, 177, 179, 180, 181, 183, 185, 187, 189, 190, 194, 195, 196 203, 205, 206. Analysis restricted to women becoming pregnant while under observation in the prospective cohort. Cox proportional hazards regression. adjusted for: age (24 - 30 versus 30 - 34) and mean alcohol consumption and cigarette smoking standardized to a 28-day menstrual cycle.

genic PCB tertile was not positively associated with pregnancy loss when compared with the lowest estrogenic PCB tertile $(\mathrm{aHR}=0.86,95 \% \mathrm{CI}$ : $0.23,3.11)$. When continuous estrogenic, anti-estrogenic, and other PCBs and DDE were jointly included in the model, estrogenic PCBs $(\mathrm{aHR}=1.77,95 \% \mathrm{CI}: 0.34,9.25)$ and
DDE $(\mathrm{aHR}=1.68,95 \% \mathrm{CI}: 0.50,5.61)$ yielded positive point estimates and wide confidence intervals that included the null (Table 4). We conducted sensitivity analyses first restricting the models to women with complete exposure data (no imputations) and then substituting log transformed PCB and DDE values for the raw 
Table 4. Hazard of pregnancy loss and serum concentration of polychlorinated biphenyl (PCBs) and 1,1-dichloro-2,2-bis (p-chlorophenyl)ethylene (DDE) analyzed jointly $(n=69)$.

\begin{tabular}{ccc}
\hline Serum Concentration (ng/g serum) & Unadjusted HR $(95 \% \mathrm{CI})$ & ${\text { Adjusted HR }(95 \% \mathrm{CI})^{\mathrm{a}}}^{\mathrm{a}}$ \\
\hline Estrogenic PCBs & $1.64(0.29,9.36)$ & $1.77(0.34,9.25)$ \\
Anti-estrogenic PCBs & $0.02(<0.01,196.01)$ & $0.09(<0.01,632.71)$ \\
Other PCBs & $0.96(0.18,5.09)$ & $0.91(0.19,4.41)$ \\
DDE & $1.38(0.44,4.39)$ & $1.68(0.50,5.61)$ \\
\hline
\end{tabular}

NOTE: Estrogenic PCBs includes $\sum$ congeners 4_10, 5_8, 15_17, 18, 31, 44, 47, 48, 52, 70, 99, 101, 136, 153, 188; anti-estrogenic PCBs includes $\sum$ congeners $77110,105,114,126,156171,169$; and other includes $\sum$ PCB congeners $6,79,1632,19,22,2427,25,28,33,40,42,45,50,55,59,60,64,6695,74$, $8187,82,94,97,118,128,129,132,134,135,138,141,147,149,151,157 \_200,163,167,170,172,174,176,177,179,180,181,183,185,187,189,190,194$, $19 \overline{5}, 196 \_203,205,206$. Analysis restricted to women becoming pregnant while under observation in the prospective cohort. Cox proportional hazards regression. adjusted for: age (24 - 30 versus 30 - 34) and mean alcohol consumption and cigarette smoking standardized to a 28-day menstrual cycle.

measurements and observed similar results (data not shown).

\section{Discussion}

Our prospective pregnancy study with preconception enrollment of women and a sensitive hCG biomarker of pregnancy did not find statistically significant associations between DDE, total, estrogenic, and anti-estrogenic PCBs, and pregnancy loss. The point estimates for anti-estrogenic PCBs were less than one, possibly suggesting a different direction of effect, but the estimate was imprecise. One possible explanation for the suggestion of a protective effect on the hazard of pregnancy loss for anti-estrogenic PCBs may be attributed to the concept of competing risk. If an exposure reduces pregnancy likelihood, fewer losses would be expected, given that preg-nancy is a necessary criterion for a loss. Anti-estrogenic PCB concentrations were associated with a reduction in fecundability or a longer time to pregnancy for this cohort [25]. In this cohort, 6- and 12-month cumulative pregnancies were $76 \%$ and $94 \%$, which are similar to the two other prospective pregnancy studies conducting 12 month follow-up of couples. Cumulative 6-and 12-cycle pregnancy rates for other prospective cohort studies following women through 12 cycles ranged from $81 \%$ 90\% [36] and 92\% - 95\% [37].

Another explanation may be attributed to the laboratory precision in measuring the anti-estrogenic PCB congeners or the absence of uniform criteria for grouping congeners with regard to biologic activity. The suggestion of opposing directions for our point estimates by congener grouping supports the need to more fully assess the mixture of compounds in relation to sensitive reproductive and developmental outcomes, particularly in preconception cohort studies with ample power for the detection of pregnancy losses.

The only other prospective cohort study that utilized preconception enrollment of women, enabling the au- thors to identify incident pregnancy losses found a positive association between pregnancy loss and DDT [18]. Specifically, 388 nulliparous Chinese women provided a blood specimen for the quantification of serum DDE and daily urine samples for up to one year for the detection of hCG pregnancy. An increased odds ratio (OR) for early pregnancy loss of 1.17 (95\% CI: 1.05, 1.29) was associated with a $10-\mathrm{ng} / \mathrm{g}$ increase in serum total DDT, and in a categorical analysis comparing the third tertile of exposure to the first, found an adjusted OR 2.12 (95\% CI: $1.26,3.57)$ [18]. Concentrations were notably higher among the Chinese women (median $p, p^{\prime}$-DDE and total DDT of $26.24 \mathrm{ng} / \mathrm{g}$ and 27.9, respectively) than in our study (median DDE $0.97 \mathrm{ng} / \mathrm{g}$ ), possibly reflecting the more recent use of DDT in China compared to the United States [38]. Increased odds of spontaneous abortion have been associated for DDE in a subset of the US Collaborative Pregnancy Cohort, which recruited already pregnant women at median gestational age 21 weeks [14,39]. A small case control study of 30 Chinese factory workers also reported that $p, p^{\prime}$-DDE was associated with a 1.13 (CI: 1.02, 1.26) increased odds of spontaneous pregnancy loss [13]. Lastly, support for an association between PCB and/or DDE exposure and pregnancy loss comes from several studies that relied upon retrospectively reported exposures or outcomes and often without added attention to the role of lactation history in mediating effects [40-42]. Women with pregnancy losses have less opportunity to decrease their body burden of PCBs and DDE than women giving birth, especially if they opt to breastfed. Retrospective exposures that rely on complex decay models for estimating exposures at some time in the past such as during pregnancy may be a source of potential exposure misclassification [43]. Our results are not inconsistent with the existing literature on DDE and are subject to limited power, though the direction of effect, particularly for DDE, signals a similar direction of effect as prior work. 
The mechanism of action by which DDE interferes with pregnancy maintenance is not known, but DDE isomers are believed to act similarly to estradiol $[44,45]$ and, thereby, may interfere with endocrine function. Alternatively, DDE and PCBs have been demonstrated to induce cytochrome $\mathrm{P} 450$ hepatic enzymes, which could interfere with normal hormonal production necessary for pregnancy maintenance $[46,47]$ or the metabolism of other xenobiotics, which may enhance or diminish hormonal or biochemical processes $[48,49]$. Further, persistent organochlorine compounds may disrupt the placental cell sodium channel barrier and, thereby, affect progesterone receptor binding [14].

\section{Conclusions}

We sought to assess a mixture of persistent organochlorine compounds in relation to impaired fecundity, particularly the risk of pregnancy loss, to better model human exposures by addressing biological mechanism of action. The use of daily diaries for ascertaining lifestyle exposures believed relevant for human fecundity is advantageous, since women reported lifestyle exposures prior to knowledge of pregnancy outcome. We systematically ascertained pregnancy via sensitive home pregnancy test kits, and we know no a priori reason why test results would vary by exposure status. Our findings await replication from larger cohort studies with preconception enrollment and longitudinal data collection with the eventual expectation of filling critical data gaps regarding the impact of persistent compounds on sensitive markers of human reproduction and development.

Our findings are not inconsistent with prior findings of an association between DDE and estrogenic PCBs and pregnancy loss. Though limited by a lack of power, our results underscore the value of efforts to measure chemical mixtures and better approximate the biological mode of action. Further, due to preconceptional enrollment of women and the pregnancy testing protocol, there is very little possibility for missed pregnancy losses.

\section{Acknowledgements}

This research was supported by the Intramural Research Program of the Eunice Kennedy Shriver National Institute of Child Health and Human Development, National Institutes of Health.

\section{REFERENCES}

[1] J. M. Wood, "Dynamics of Human Reproduction: Biology, Biometry, Demography." Aldine de Gruyter, New York, 1994.

[2] C. Gini, "Premieres Recheres Sur la Fecondabilite de la Femme," Proceedings of the International Mathematical
Conference, Vol. 2, 1924, pp. 889-892.

[3] A. J. Wilcox, C. R. Weinberg, J. F. O'Connor, D. D. Baird, J. P. Schlatterer, R. E. Canfield, et al., "Incidence of Early Loss of Pregnancy," New England Journal of Medicine, Vol. 319, No. 4, 1988, pp. 189-194. doi:10.1056/NEJM198807283190401

[4] G. P. Daston, J. W. Gooch, W. J. Breslin, et al., "Environmental Estrogens and Reproductive Health: A Discussion of the Human and Environmental Data," Reproductive Toxicology, Vol. 11, No. 4, 1997, pp. 465-481. doi:10.1016/S0890-6238(97)00014-2

[5] E. Diamanti-Kandarakis, J. P. Bourguignon, L. C. Giudice, R. Hauser, G. S. Prins, A. M. Soto, et al., "Endocrine-Disrupting Chemicals: An Endocrine Society Scientific Statement," Endocrine Reviews, Vol. 30, No. 4, 2009, pp. 293-342. doi:10.1210/er.2009-0002

[6] G. Toft, L. Hagmar, A. Giwercman and J. P. Bonde, "Epidemiological Evidence on Reproductive Effects of Persistent Organochlorines in Humans," Reproductive Toxicology, Vol. 19, No. 1, 2004, pp. 5-26. doi:10.1016/i.reprotox.2004.05.006

[7] T. M. Crisp, E. D. Clegg, R. L. Cooper, W. P. Wood, D. G. Anderson, K. P. Baetcke, et al., "Environmental Endocrine Disruption: An Effects Assessment and Analysis," Environ Health Perspect, Vol. 106, Supplement 1, 1998, pp. 11-56.

[8] L. G. M. Buck, C. D. Lynch and M. A. Cooney, "Environmental Influences on Female Fecundity and Fertility," Seminars in Reproductive Medicine, Vol. 24, No. 3, 2006, pp. 147-155. doi:10.1055/s-2006-944421

[9] P. Mendola, G. M. Buck, J. E. Vena, M. Zielezny and L. E. Sever, "Consumption of PCB-Contaminated Sport Fish and Risk of Spontaneous Fetal Death," Environ Health Perspect, Vol. 103, No. 5, 1995, pp. 498-502. doi:10.1289/ehp.95103498

[10] A. Axmon, L. Rylander, U. Stromberg and L. Hagmar, "Miscarriages and Stillbirths in Women with a High Intake of Fish Contaminated with Persistent Organochlorine Compounds," International Archives of Occupational and Environmental Health, Vol. 73, No. 3, 2000, pp. 204-208. doi: $10.1007 / \mathrm{s} 004200050028$

[11] K. Hemminki, M. L. Niemi, I. Saloniemi, H. Vainio and E. Hemminki, "Spontaneous Abortions by Occupation and Social Class in Finland," International Journal of Epidemiology, Vol. 9, No. 2, 1980, pp. 149-153. doi:10.1093/ije/9.2.149

[12] D. O. Carpenter, Y. Shen, T. Nguyen, L. Le and L. L. Lininger, "Incidence of Endocrine Disease among Residents of New York Areas of Concern," Environmental Health Perspectives, Vol. 109, Supplement 6, 2001, pp. 845-851. doi: $10.2307 / 3454646$

[13] S. A. Korrick, C. Chen, A. I. Damokosh, J. Ni, X. Liu, S. I. Cho, et al., "Association of DDT with Spontaneous Abortion: A Case-Control Study," Annals of Epidemiology, Vol. 11, No. 7, 2001, pp. 491-496. doi:10.1016/S1047-2797(01)00239-3

[14] M. P. Longnecker, M. A. Klebanoff, D. B. Dunson, X. 
Guo, Z. Chen, H. Zhou, et al., "Maternal Serum Level of the DDT Metabolite DDE in Relation to Fetal Loss in Previous Pregnancies," Environmental Research, Vol. 97, No. 2, 2005, pp. 127-133. doi:10.1016/S0013-9351(03)00108-7

[15] A. M. Sweeney, M. R. Meyer, J. H. Aarons, J. L. Mills and R. E. LaPorte, "Evaluation of Methods for the Prospective Identification of Early Fetal Losses in Environmental Epidemiology Studies," American Journal of Epidemiology, Vol. 127, No. 4, 1988, pp. 843-850.

[16] R. E. Chapin, W. A. Robbins, L. A. Schieve, A. M. Sweeney, S. A. Tabacova and K. M. Tomashek, "Off to a Good Start: The Influence of Pre- and Periconceptional Exposures, Parental Fertility, and Nutrition on Children's Health," Environmental Health Perspectives, Vol. 112, No. 1, 2004, pp. 69-78. doi:10.1289/ehp.6261

[17] G. M. Buck, C. D. Lynch, J. B. Stanford, A. M. Sweeny, L. A. Schieve, J. C. Rockett, et al., "Prospective Pregnancy Study Designs for Assessing Reproductive and Developmental Toxicants," Environmental Health Perspectives, Vol. 112, No. 1, 2004, pp. 79-86. doi:10.1289/ehp.6262

[18] S. A. Venners, S. Korrick, X. Xu, C. Chen, W. Guang, A. Huang, et al., "Preconception Serum DDT And Pregnancy Loss: A Prospective Study Using A Biomarker Of Pregnancy," American Journal of Epidemiology, Vol. 162, No. 8, 2005, pp. 709-716. doi:10.1093/aje/kwi275

[19] M. Sugiura-Ogasawara, Y. Ozaki, S. Sonta, T. Makino and K. Suzumori, "PCBs, Hexachlorobenzene and DDE Are Not Associated with Recurrent Miscarriage," American Journal of Epidemiology, Vol. 50, No. 6, 2003, pp. 485-489.

[20] I. Gerhard, V. Daniel, S. Link, B. Monga and B. Runnebaum, "Chlorinated Hydrocarbons in Women with Repeated Miscarriages," Environmental Health Perspectives, Vol. 106, No. 10, 1998, pp. 675-681. doi:10.1289/ehp. 98106675

[21] L. A. Cole, S. A. Khanlian, J. M. Sutton, S. Davies and W. F. Rayburn, "Accuracy of Home Pregnancy Tests at the Time of Missed Menses," American Journal of Obstetrics and Gynecology, Vol. 190, No. 1, 2004, pp. 100-105. doi:10.1016/j.ajog.2003.08.043

[22] M. S. Bloom, L. G. M. Buck, E. F. Schisterman, A. Liu and P. J. Kostyniak, "Maternal Serum Polychlorinated Biphenyl Concentrations Across Critical Windows of Human Development," Environmental Health Perspectives, Vol. 115, No. 9, 2007, pp. 1320-1324. doi:10.1289/ehp.10086

[23] H. B. Greizerstein, P. Gigliotti, J. Vena, J. Freudenheim and P. J. Kostyniak, "Standardization of a Method for the Routine Analysis of Polychlorinated Biphenyl Congeners and Selected Pesticides in Human Serum and Milk," Journal of Analytical Toxicology, Vol. 21, No. 7, 1997, pp. 558-566.

[24] P. S. Cooke, T. Sato and D. L. Buchanan, "Disruption of Steroid Hormone Signaling by PCBs," In: L. W. Robertson and L. G. Hansen, Eds., PCBs: Recent Advances in
Environmental Toxicology and Health Effects, The University Press of Kentucky, Lexington, 2001, pp. 257-263.

[25] L. G. M. Buck, J. Dmochowski, C. Lynch, P. Kostyniak, B. M. McGuinness and J. E. Vena, "Polychlorinated Biphenyl Serum Concentrations, Lifestyle and Time-toPregnancy," Human Reproduction, Vol. 24, No. 2, 2009 , pp. 451-458. doi:10.1093/humrep/den373

[26] E. F. Schisterman, A. Vexler, B. W. Whitcomb and A. Liu, "The Limitations Due to Exposure Detection Limits for Regression Models," American Journal of Epidemiology, Vol. 163, No. 4, 2006, pp. 374-383. doi:10.1093/aje/kwj039

[27] D. B. Richardson and A. Ciampi, "Effects of Exposure Measurement Error When an Exposure Variable Is Constrained by a Lower Limit," American Journal of Epidemiology, Vol. 157, No. 4, 2003, pp. 355-363. doi:10.1093/aje/kwf217

[28] E. F. Schisterman, B. W. Whitcomb, G. M. Louis and T. A. Louis, "Lipid Adjustment in the Analysis of Environmental Contaminants and Human Health Risks," Environ Health Perspect, Vol. 113, No. 7, 2005, pp. 853-857. doi:10.1289/ehp.7640

[29] D. L. Phillips, J. L. Pirkle, V. W. Burse, J. T. Bernert, L. O. Henderson and L. L. Needham, "Chlorinated Hydrocarbon Levels in Human Serum: Effects of Fasting and Feeding," Archives of Environmental Contamination and Toxicology, Vol. 18, No. 4, 1989, pp. 495-500. doi:10.1007/BF01055015

[30] S. D. Holladay and R. J. Smialowicz, "Development of the Murine and Human Immune System: Differential Effects of Immunotoxicants Depend on Time of Exposure," Environmental Health Perspectives, Vol. 108, No. S3, 2000, pp. 463-473. doi:10.2307/3454538

[31] G. J. van der Heijden, A. R. Donders, T. Stijnen and K. G. Moons, "Imputation of Missing Values Is Superior to Complete Case Analysis and the Missing-Indicator Method in Multivariable Diagnostic Research: A Clinical Example," Journal of Clinical Epidemiology, Vol. 59, No. 10, 2006, pp. 1102-1109. doi:10.1016/j.jclinepi.2006.01.015

[32] K. G. Moons, R. A. Donders, T. Stijnen and F. E. Harrell, "Using the Outcome for Imputation of Missing Predictor Values Was Preferred," Journal of Clinical Epidemiology, Vol. 59, No. 10, 2006, pp. 1092-1101. doi:10.1016/j.jclinepi.2006.01.009

[33] W. Vach and M. Blettner, "Biased Estimation of the Odds Ratio in Case-Control Studies Due to the Use of Ad Hoc Methods of Correcting for Missing Values for Confounding Variables," American Journal of Epidemiology, Vol. 134, No. 8, 1991, pp. 895-907.

[34] P. P. Howards, E. F. Schisterman and P. J. Heagerty, "Potential Confounding by Exposure History and Prior Outcomes: An Example from Perinatal Epidemiology," Epidemiology, Vol. 18, No. 5, 2007, pp. 544-551. doi:10.1097/EDE.0b013e31812001e6

[35] C. R. Weinberg, "Toward a Clearer Definition of Confounding," American Journal of Epidemiology, Vol. 137, 
No. 1, 1993, pp. 1-8.

[36] C. Gnoth, D. Godehardt, E. Godehardt, P. Frank-Herrmann and G. Freundl, "Time to Pregnancy: Results of the German Prospective Study and Impact on the Management of Infertility," Human Reproduction, Vol. 18, No. 9, 2003, pp. 1959-1966. doi:10.1093/humrep/deg366

[37] X. Wang, C. Chen, L. Wang, D. Chen, W. Guang and J. French, "Conception, Early Pregnancy Loss, and Time to Clinical Pregnancy: A Population-Based Prospective Study," Fertility and Sterility, Vol. 79, No. 3, 2003, pp. 577-584. doi:10.1016/S0015-0282(02)04694-0

[38] W. J. Rogan and A. Chen, "Health Risks and Benefits of Bis(4-Chlorophenyl)-1,1,1-Trichloroethane (DDT)," Lancet, Vol. 366, No. 9487, 2005, pp. 763-773. doi:10.1016/S0140-6736(05)67182-6

[39] A. Chen, M. L. Pennell, M. A. Klebanoff, W. J. Rogan and M. P. Longnecker, "Maternal Smoking during Pregnancy in Relation to Child Overweight: Follow-up to Age 8 Years," International Journal of Epidemiology, Vol. 35, No. 1, 2006, pp. 121-130. doi:10.1093/ije/dyi218

[40] L. E. Redding, M. D. Sohn, T. E. McKone, J. W. Chen, S. L. Wang, D. P. Hsieh, et al., "Population Physiologically Based Pharmacokinetic Modeling for the Human Lactational Transfer of PCB-153 with Consideration of Worldwide Human Biomonitoring Results," Environmental Health Perspectives, Vol. 116, No. 12, 2008, pp. 16291635. Perspectives, Vol. 116, No. 12, 2008, pp. 16291635. doi:10.1289/ehp.11519

[41] H. R. Chao, S. L. Wang, C. C. Lee, H. Y. Yu, Y. K. Lu and O. Papke, "Level of Polychlorinated Dibenzo-P-Dioxins, Dibenzofurans and Biphenyls (PCDD/Fs, Pcbs) in Human Milk and the Input to Infant Body Burden," Food and Chemical Toxicology, Vol. 42, No. 8, 2004, pp. 1299-1308. doi:10.1016/j.fct.2004.03.008

[42] L. A. Gallenberg and M. J. Vodicnik, "Potential Mechanisms for Redistribution of Polychlorinated Biphenyls during Pregnancy and Lactation," Xenobiotica, Vol. 17, No. 3, 1987, pp. 299-310. doi: $10.3109 / 00498258709043940$

[43] A. Axmon, A. M. Thulstrup, A. Rignell-Hydbom, H. S.
Pedersen, V. Zvyezday, J. K. Ludwicki, et al., "Time to Pregnancy as a Function of Male and Female Serum Concentrations of 2,2'4,4'5,5'-Hexachlorobiphenyl (CB-153) and 1,1-Dichloro-2,2-Bis (P-Chlorophenyl)-Ethylene (p,p'DDE)," Human Reproduction, Vol. 21, No. 3, 2006, pp. 657-665. doi:10.1093/humrep/dei397

[44] J. S. Ireland, V. R. Mukku, A. K. Robison and G. M. Stancel, "Stimulation of Uterine Deoxyribonucleic Acid Synthesis by 1,1,1-Trichloro-2-(p-Chlorophenyl)-2-(oChlorophenyl)ethane (o,p'-DDT)," Biochemical Pharmacology, Vol. 29, No. 11, 1980, pp. 1469-1474. doi:10.1016/0006-2952(80)90596-1

[45] G. M. Stancel, J. S. Ireland, V. R. Mukku and A. K. Robison, "The Estrogenic Activity of DDT: In Vivo and in Vitro Induction of a Specific Estrogen Inducible Uterine Protein by o,p'-DDT," Life Science, Vol. 27, No. 12, 1980, pp. 1111-1117. doi:10.1016/0024-3205(80)90037-5

[46] R. W. Nims, R. A. Lubet, S. D. Fox, P. E. Thomas, A. B. Reddy, T. A. Kocarek, et al., "Comparative Pharmacodynamics of CYP2B Induction by DDT, DDE, and DDD in Male Rat Liver and Cultured Rat Hepatocytes," Journal of Toxicology and Environmental Health, Part A, Vol. 53, No. 6, 1998, pp. 455-477.

doi: $10.1080 / 009841098159187$

[47] R. W. Nims and R. A. Lubet, "Induction of Cytochrome P-450 in the Norway Rat, Rattus Norvegicus, Following Exposure to Potential Environmental Contaminants," Journal of Toxicology and Environmental Health, Vol. 46, No. 3, 1995, pp. 271-292. doi:10.1080/15287399509532035

[48] M. S. Petersen, J. Halling, P. Damkier, N. Flemming, P. Grandjean, P. Weihe, et al., "Polychlorinated Biphenyl (PCB) Induction of CYP3A4 Enzyme Activity in Healthy Faroese Adults," Toxicology and Applied Pharmacology, Vol. 224, No. 2, 2007, pp. 202-206. doi:10.1016/j.taap.2007.07.002

[49] F. P. Guengerich, D. H. Kim and M. Iwasaki, "Role of human cytochrome P-450 IIE1 in the oxidation of many low molecular weight cancer suspects," Chemical Research in Toxicology, Vol. 4, No. 2, 1991, pp. 168-179. doi: $10.1021 / \mathrm{tx} 00020 \mathrm{a} 008$ 